\title{
Kernels of L-functions and shifted convolutions
}

\author{
Nikolaos Diamantis (University of Nottingham) \\ nikolaos.diamantis@nottingham.ac.uk
}

\section{Introduction}

Many of the outstanding questions on values of $L$-functions associated to cusp forms and values of their derivatives are subsumed under major conjectures of number theory such as those of Birch-Swinnerton-Dyer, Deligne, Beilinson, Bloch-Kato etc. For example, an important part of Beilinson's conjecture reveals the algebraic nature of values of $L$-functions and their derivatives in terms of periods.

In the interpretation of Kontsevich-Zagier ([15]), periods form a class $\mathcal{P}$ of numbers which are arithmetically significant without necessarily being algebraic. Specifically, a complex number is called a period if its real and imaginary parts are absolutely convergent integrals of rational functions over $\mathbb{Q}$ with a domain of integration defined by polynomial inequalities over $\mathbb{Q}$, e.g.

$$
\pi=\iint_{x^{2}+y^{2} \leq 1} d x d y \quad \text { and } \log (n)=\int_{1}^{n} \frac{1}{x} d x(\text { for } n \in \mathbb{N}) .
$$

With this terminology, we can state a part of Beilinson's conjecture in a version given in [15].

Conjecture. ([15]) Let $f$ be a weight $k$ Hecke eigencuspform for $S L_{2}(\mathbb{Z}), L_{f}(s)$ its L-function, and $m$ an integer. Then, if $r$ is the order of vanishing of $L_{f}(s)$ at $s=m$, we have

$$
L^{(r)}(m) \in \mathcal{P}[1 / \pi] .
$$

Although this conjecture is open in general, some cases have been settled, e.g. the case $r=0$, corresponding to values of $L$-functions (see $[10,5,1]$ etc. or [15]).

However, even for $r=0$, it turns out that other characterisations of the values of $L$ functions are possible and important (besides their property of being periods). For instance,

i) Given that $L_{f}(n)(n \in \mathbb{Z})$ are periods (up to a power of $\pi$ ), it is interesting to find explicit expressions for values of $L$-functions and values of their derivatives as integrals, in accordance with the above definition of periods. Methods to address this question for specific L-functions have been developed in $[3,13]$ etc.

ii) In [2], non-critical values of $L$-functions (i.e. the case $r=0$ and $m \geq k$ ) have been expressed in terms of multiple modular values. This connection emerged in a vastly general algebro-geometric context which was driven by fundamental questions in the theory of motives. 
iii) A characterisation of the field containing an arbitrary value of $L_{f}(s)$ has been established in [7]. It involves certain double Eisenstein series and applies even to $s$ that are not integers.

In this note we characterise ratios of non-critical values of $L$-functions based on a combination of the methods of ii) and iii) together with a new element, namely shifted divisor sum Dirichlet series. This Dirichlet series is given, in its domain of initial convergence, by

$$
D_{h}(\alpha, \beta ; s):=\sum_{\substack{n \in \mathbb{N} \\ n>h}} \frac{\sigma_{\alpha}(n) \sigma_{\beta}(n-h)}{n^{s}}, \quad \alpha, \beta, s \in \mathbb{C}, h \in \mathbb{N} .
$$

It has recently been studied, in a slightly different form, by M. Kiral who showed [8] that it has meromorphic continuation to the entire $\mathbb{C}$.

Our main result then is as follows.

Theorem 1.1. Let $f$ be a normalized weight $k$ cuspidal eigenform for $\Gamma:=S L_{2}(\mathbb{Z})$ and let $L_{f}^{*}(s)$ be its completed L-function.

Denote by $\mathcal{D}_{m}$ the field generated over $\mathbb{Q}(\pi i)$ by

$$
\begin{gathered}
D_{l}(k+m-2,-m-2 ; n),(n=k-2, \ldots, k-2+m ; l \in \mathbb{N}), \quad \text { if } m \text { is odd, and by } \\
\quad D_{l}(k+m-1,-m-1 ; n),(n=k-1, \ldots, k-1+m ; l \in \mathbb{N}), \quad \text { if } m \text { is even. }
\end{gathered}
$$

Also, let $\mathbb{Q}(f)$ be the field generated by the Fourier coefficients of $f$.

Then, for each integer $r \geq 1$ we have

$$
\frac{L_{f}^{*}(k+r)}{L_{f}^{*}(k+1)} \in \mathcal{D}_{r} \mathcal{D}_{1} \mathbb{Q}(f) \text { if } r \text { is odd and } \frac{L_{f}^{*}(k+r)}{L_{f}^{*}(k+2)} \in \mathcal{D}_{r} \mathcal{D}_{2} \mathbb{Q}(f) \text { if } r \text { is even. }
$$

It should be stressed that the fields in the statement of the theorem are independent of the cusp form $f$. It is not clear if a much more precise description of that field would be possible. For example, in [9] it is actually proved that non-critical values cannot all belong to a finitely generated $\mathbb{Z}$-module. Other descriptions, e.g. in terms of finite transcedence degree, while not disproved, do not seem to be anticipated by the main conjectures. It should also be noted that we do not know yet how the fields $\mathcal{D}_{r}$ are exactly related to Zagier's periods.

We were led to Theorem 1.1 by an approach based on comparing the kernels behind characterisations ii) and iii) above. The former was a real-analytic kernel of $L_{f}^{*}(s) L_{f}^{*}(w)$ given by $\mathrm{F}$. Brown for certain pairs of integers $s, w([2])$. The latter was a kernel function, also for $L_{f}^{*}(s) L_{f}^{*}(w)$ but for all $s, w \in \mathbb{C}$, which was holomorphic and had the form of a double Eisenstein series $\mathbf{E}_{s}^{*}([7])$.

This approach has several applications and here we present two of them: First, Prop. 2.3 gives a characterisation of the ratio of the "transcendental factors" in Manin's Theorem [10], whose nature has not been well understood. The second application is Theorem 1.1 which answers a question left unanswered in [7], namely to interpret the Fourier coefficients of $\mathbf{E}_{s}^{*}$ (and, through them, the ratios of L-values) by recognisable quantities. Here we prove that these quantities belong to the class of shifted divisor sum convolutions series. Elements of this class are the focus of intense investigation by various methods because of their natural 
appearance in moment and other analytic problems. Shifted divisor sum convolutions, in particular, are important for the binary additive divisor problem, for example.

It should be noted that, for the computation leading to Th. 1.1, the use of $\mathbf{E}_{s}^{*}$ could be avoided but we maintain it in the arrangement of the proof presented here because it allows a more unified treatment of ratios of L-functions that works even in the cases not covered by the construction of [2], e.g. in the setting of Prop. 2.3.

Acknowledgement. The author thanks the referee for a very careful reading of the manuscript and for many useful comments and suggestions.

\section{The field of L-values}

Let $f$ be a weight $k$ cuspidal eigenform

$$
f(z)=\sum_{n=1}^{\infty} a(n) e^{2 \pi i n z}
$$

for $\mathrm{SL}_{2}(\mathbb{Z})$ normalised so that $a(1)=1$. Let $L_{f}(s)$ denote its L-function and consider its completed version

$$
L_{f}^{*}(s)=(2 \pi)^{-s} \Gamma(s) L_{f}(s) .
$$

We will now define the double Eisenstein series introduced in [7]. Set $B=\left\{\left(\begin{array}{ll}1 & n \\ 0 & 1\end{array}\right) ; n \in \mathbb{Z}\right\}$. Also set,

$$
c_{\gamma}:=c \quad \text { and } j(\gamma, z)=c z+d \quad \text { for } \quad\left(\begin{array}{ll}
* * \\
c & d
\end{array}\right) \in \mathrm{SL}_{2}(\mathbb{Z}) .
$$

With the convention $-\pi<\arg (z) \leq \pi$ define

$$
\mathbf{E}_{s}(z, w):=\sum_{\substack{\gamma, \delta \in B \backslash \Gamma \\ c_{\gamma \delta}-1>0}}\left(c_{\gamma \delta^{-1}}\right)^{w-1}\left(\frac{j(\gamma, z)}{j(\delta, z)}\right)^{-s} j(\delta, z)^{-k}
$$

As shown in [7], this series can be thought of as a Rankin-Cohen bracket of not necessarily integer index, applied to pair of Eisenstein series. We also define the completed double Eisenstein series

$$
\mathbf{E}_{s}^{*}(z, w):=\frac{\Gamma(s) \Gamma(k-s) \Gamma(k-w) \zeta(1-w+s) \zeta(1-w+k-s)}{e^{-s i \pi / 2} 2^{3-w} \pi^{k+1-w} \Gamma(k-1)} \mathbf{E}_{s}(z, w) .
$$

In [7] it is proved:

Theorem 2.1. The series $\mathbf{E}_{s}^{*}(z, w)$ converges absolutely and uniformly on compact sets for which $2<\operatorname{Re}(s)<k-2$ and $\operatorname{Re}(w)<\min (\operatorname{Re}(s)-1, k-1-\operatorname{Re}(s))$. It has an analytic continuation to all $s, w \in \mathbb{C}$ and, as a function of $z$, it is a weight $k$ cusp form for $\Gamma$. We have

$$
\left\langle\mathbf{E}_{s}^{*}(\cdot, w), f\right\rangle=L_{f}^{*}(s) L_{f}^{*}(w)
$$

for any normalised cuspidal eigenform $f$ of weight $k$. 
With this theorem, we characterise the field of values of $L_{f}^{*}(s)$ using a method motivated by Zagier's technique ([17]). His technique is based on standard (positive integer index) RankinCohen brackets. We will state and prove a slightly more general version of the characterisation given in [7].

For cusp forms $f_{1}, f_{2}, \ldots$ we will be denoting by $\mathbb{Q}\left(f_{1}, f_{2}, \ldots\right)$ the field obtained by adjoining to $\mathbb{Q}$ the Fourier coefficients of $f_{1}, f_{2} \ldots$

Proposition 2.2. Let $f$ be a normalised cuspidal eigenform $f$ of weight $k$ and let $s_{0} \in \mathbb{C}$ such that $L_{f}^{*}\left(s_{0}\right) \neq 0$. Then, for all $s, w \in \mathbb{C}$, with $L_{f}^{*}(s) \neq 0$,

$$
\frac{L_{f}^{*}(w)}{L_{f}^{*}(s)} \in \mathbb{Q}\left(\mathbf{E}_{s_{0}}^{*}(\cdot, w), \mathbf{E}_{s_{0}}^{*}(\cdot, s), f\right) .
$$

Proof. With Th. 2.1 we have

$$
\frac{L_{f}^{*}(w)}{L_{f}^{*}(s)}=\frac{L_{f}^{*}\left(s_{0}\right) L_{f}^{*}(w)}{L_{f}^{*}\left(s_{0}\right) L_{f}^{*}(s)}=\frac{\left\langle\mathbf{E}_{s_{0}}^{*}(\cdot, s), f\right\rangle}{\left\langle\mathbf{E}_{s_{0}}^{*}(\cdot, w), f\right\rangle}=\frac{\left\langle\mathbf{E}_{s_{0}}^{*}(\cdot, s), f\right\rangle /\langle f, f\rangle}{\left\langle\mathbf{E}_{s_{0}}^{*}(\cdot, w), f\right\rangle /\langle f, f\rangle}
$$

By a general result (see, e.g. [14], Lemma 4), the numerator of the last fraction belongs to $\mathbb{Q}\left(\mathbf{E}_{s_{0}}^{*}(\cdot, s), f\right)$. Likewise for the denominator. This implies the result.

We state a result which can be deduced from this proposition.

According to Manin's Periods Theorem [10] there are $\omega^{+}(f), \omega^{-}(f) \in \mathbb{R}$ such that

$$
L_{f}^{*}(s) / \omega^{+}(f) \in \mathbb{Q}(f) \quad \text { and } L_{f}^{*}(w) / \omega^{-}(f) \in \mathbb{Q}(f)
$$

for all $s, w$ with $1 \leq s, w \leq k-1$ and $s$ even, $w$ odd. Although we know that $\omega^{ \pm}(f)$ are periods (cf. Sect. 3.4 of [15]) and that (when appropriately normalised) their product is $\langle f, f\rangle$, little is known about their quotient. However, Prop. 2.2 implies the following characterisation of the field to which their quotient belongs.

Proposition 2.3. Let $f$ be a normalised cuspidal eigenform of weight $k$ for $S L_{2}(\mathbb{Z})$ such that $L_{f}^{*}(k / 2) \neq 0$. (In particular, $k \equiv 0 \bmod 4$. ) Then

$$
\frac{\omega^{+}(f)}{\omega^{-}(f)} \in \mathbb{Q}\left(\pi, i, \mathbf{E}_{k / 2}^{*}(\cdot, 4), f\right)
$$

Proof. Set $w=4, s=3$ and $s_{0}=k / 2$ in Prop. 2.2. Since $L_{f}^{*}(4) / \omega^{+}(f), L_{f}^{*}(3) / \omega^{-}(f) \in \mathbb{Q}(f)$, we deduce

$$
\frac{\omega^{+}(f)}{\omega^{-}(f)} \in \mathbb{Q}\left(\mathbf{E}_{k / 2}^{*}(\cdot, 3), \mathbf{E}_{k / 2}^{*}(\cdot, 4), f\right) .
$$

With Prop. 2.4 of [7],

$$
\mathbf{E}_{k / 2}^{*}(\cdot, 3)=\mathbf{E}_{\left(\frac{k}{2}-2\right)+2}^{*}(\cdot, 2+1)
$$

equals (up to an factor in $\mathbb{Q}(\pi, i)$ ) the Rankin-Cohen bracket

$$
\left[E_{k / 2}, E_{k / 2}\right]_{2}:=\sum_{r=0}^{2}(-1)^{r}\left(\begin{array}{c}
\frac{k}{2}+1 \\
2-r
\end{array}\right)\left(\begin{array}{c}
\frac{k}{2}+1 \\
r
\end{array}\right) E_{k / 2}^{(r)} E_{k / 2}^{(2-r)}
$$


where

$$
E_{2 m}(z)=\sum_{\gamma \in \Gamma_{\infty} \backslash \Gamma} j(\gamma, z)^{-2 m}=-\frac{B_{2 m}}{4 m}+\sum_{n=1}^{\infty} \sigma_{2 m-1}(n) e^{2 \pi i n z} .
$$

From the general theory of Rankin-Cohen brackets, $\left[E_{k / 2}, E_{k / 2}\right]_{2}$ is a cusp from, and it is clear that it has rational Fourier coefficients (see, e.g. Section 5 of [17])

Remark. In [16] (5.13), it is shown that, in a different setting (odd weight $k$ and higher level of the group), this quotient does have a simple explicit expression.

\section{Brown's kernel}

We maintain the notation of the previous section. In Section 9 of [2], F. Brown gives a kernel for a certain product of values of $L_{f}(s)$. We summarise the part of the construction we will need.

Let $i, j \geq 0$ be integers and let $s$ be such that $i+j+2 \operatorname{Re}(s)>2$. For $z=x+i y$ set

$$
\mathcal{E}_{i, j}^{s}(z)=\frac{1}{2} \sum_{\gamma \in B \backslash \Gamma} \frac{y^{s}}{j(\gamma, z)^{i+s} j(\gamma, \bar{z})^{j+s}} .
$$

This series converges absolutely in the indicated region and satisfies

$$
\mathcal{E}_{i, j}^{s}(\gamma z)=j(\gamma, z)^{i} j(\gamma, \bar{z})^{j} \mathcal{E}_{i, j}^{s}(z) \quad \text { for all } \gamma \in \Gamma \text { and } z \in \mathfrak{H} \text {. }
$$

It further has a meromorphic continuation to the entire complex plane since

$$
\mathcal{E}_{i, j}^{s}(z)=y^{-\frac{i+j}{2}} E_{i-j}\left(s+\frac{i+j}{2}\right)(z)
$$

where $E_{m}(s)(z)$ stands for the weight $m$ Eisenstein series

$$
E_{m}(s)(z)=\sum_{\gamma \in \Gamma_{\infty} \backslash \Gamma} \operatorname{Im}(\gamma z)^{s}\left(\frac{|j(\gamma, z)|}{j(\gamma, z)}\right)^{m}
$$

Here $\Gamma_{\infty}$ is the stabiliser of $\infty$.

For $m \in \mathbb{Z}_{+}$let $\langle\cdot, \cdot\rangle_{m}$ be the pairing on real-analytic functions whose product vanishes exponentially at $\infty$ which is given by the formula of the Petersson scalar product in weight $m$ :

$$
\langle g, h\rangle_{m}=\int_{\mathfrak{F}} g(z) \overline{h(z)} y^{m} \frac{d x d y}{y^{2}}
$$

where $\mathfrak{F}$ is a fundamental domain of $\mathrm{SL}_{2}(\mathbb{Z})$. Notice that strictly speaking this is not the standard Petersson scalar product because we do not require $g, h$ to be $\Gamma$-invariant.

Corollary 9.14 of [2] implies: 
Theorem 3.1. [2] Let $r \geq 1$ be an integer and let $a, b \geq 2$ be integers such that

$$
k=2 a+2 b-2 r-2 .
$$

Then for each normalised cuspidal eigenform $f$ of weight $k$ and for each $s \in \mathbb{C}$, we have

$$
\begin{aligned}
\frac{\pi^{2 a-s-k-r}}{2^{1-2 a}} \Gamma(s+k+r-2 a) \zeta(2 s+k+2 r-2 a) & \left\langle E_{2 a} \mathcal{E}_{k+r-2 a, r}^{s}, f\right\rangle_{k+r} \\
& =L_{f}^{*}(s+k+r-1) L_{f}^{*}(s+k+r-2 a)
\end{aligned}
$$

Using this construction from [2], we can now combine it with the kernel $\mathbf{E}^{*}$ of [7] to derive a key relation between Petersson scalar products.

Specifically, Theorem 3.1 combined with Theorem 2.1 implies

Proposition 3.2. For each $s \in \mathbb{C}$ and for all cusp forms of weight $k=2 a+2 b-2 r-2$ $(a, b, r \in \mathbb{N}$ and $a, b \geq 2)$ we have

$$
\begin{aligned}
\frac{\pi^{2 a-s-k-r}}{2^{1-2 a}} \Gamma(s+k+r-2 a) \zeta(2 s+k+2 r-2 a)\left\langle E_{2 a}\right. & \left.\mathcal{E}_{k+r-2 a, r}^{s}, f\right\rangle_{k+r} \\
& =\left\langle\mathbf{E}_{s+k+r-1}^{*}(z, s+k+r-2 a), f\right\rangle_{k} .
\end{aligned}
$$

This means that $\mathbf{E}_{s+k+r-1}^{*}(z, s+k+r-2 a)$ is a holomorphic projection of

$$
\frac{\pi^{2 a-s-k-r}}{2^{1-2 a}} \Gamma(s+k+r-2 a) \zeta(2 s+k+2 r-2 a) E_{2 a} \mathcal{E}_{k+r-2 a, r}^{s}
$$

in the precise sense of equation (8).

\section{Fourier coefficients}

In this section we will exploit the fact that both Th. 2.1 and Th. 3.1 give a kernel of $L_{f}^{*}(s) L_{f}^{*}(w)$ in order to compute the Fourier coefficients of $\mathbf{E}_{s+k+r-1}^{*}(z, s+k+r-2 a)$. This will then allow us to apply Prop. 2.2 to deduce Theorem 1.1.

Firstly, by the formula for Fourier coefficients of a cusp form in terms of inner products against Poincaré series, (8) implies that

the $l$-th Fourier coefficient of $\mathbf{E}_{s+k+r-1}^{*}(z, s+k+r-2 a)=$

$$
\frac{\pi^{2 a-s-k-r}(4 \pi l)^{k-1}}{2^{1-2 a} \Gamma(k-1)} \Gamma(s+k+r-2 a) \zeta(2 s+k+2 r-2 a)\left\langle E_{2 a} \mathcal{E}_{k+r-2 a, r}^{s}, P_{l}\right\rangle_{k+r}
$$

where

$$
P_{l}(z)=\sum_{\gamma \in \Gamma_{\infty} \backslash \Gamma} \frac{e^{2 \pi i l \gamma z}}{j(\gamma, z)^{k}}
$$

is the $l$-th Poincaré series of weight $k$. 
Therefore, the determination of the field of L-values appearing in Theorem 2.2 reduces to the computation of the inner products

$$
\left\langle E_{2 a} \mathcal{E}_{k+r-2 a, r}^{s}, P_{l}\right\rangle_{k+r}
$$

We will compute this integral using the Rankin-Selberg method. However, we have to modify slightly the method because otherwise, along the way we obtain a series which cannot be interchaged with integration. To overcome this difficulty we, essentially, use the "Hecke trick". Let

$$
P_{l}^{t}(z):=\sum_{\gamma \in \Gamma_{\infty} \backslash \Gamma} \operatorname{Im}(\gamma z)^{\bar{t}} \frac{e^{2 \pi i l \gamma z}}{j(\gamma, z)^{k}}
$$

is the $l$-th non-holomorphic Poincaré series of weight $k$. It is clear that

$$
\left\langle E_{2 a} \mathcal{E}_{k+r-2 a, r}^{s}, P_{l}\right\rangle_{k+r}=\left.\left\langle E_{2 a} \mathcal{E}_{k+r-2 a, r}^{s}, P_{l}^{t}\right\rangle_{k+r}\right|_{t=0} .
$$

Let $\operatorname{Re}(t) \gg 0$. With (4) we now have

$$
\begin{aligned}
\left\langle E_{2 a} \mathcal{E}_{k+r-2 a, r}^{s}, P_{l}^{t}\right\rangle_{k+r}=\int_{\mathfrak{F}} \sum_{\gamma \in \Gamma_{\infty} \backslash \Gamma} \frac{E_{2 a}(\gamma z)}{j(\gamma, z)^{2 a}} \frac{\mathcal{E}_{k+r-2 a, r}^{s}(\gamma z)}{j(\gamma, z)^{k+r-2 a} j(\gamma, \bar{z})^{r}} \operatorname{Im}(\gamma z)^{t} \frac{\overline{e^{2 \pi i l \gamma z}}}{j(\gamma, \bar{z})^{k}} y^{k+r} d \mu z \\
\quad=\int_{\mathfrak{F}} \sum_{\gamma \in \Gamma_{\infty} \backslash \Gamma} E_{2 a}(\gamma z) \mathcal{E}_{k+r-2 a, r}^{s}(\gamma z) \operatorname{Im}(\gamma z)^{t+k+r} \overline{e^{2 \pi i l \gamma z}} d \mu \gamma z .
\end{aligned}
$$

Since $E_{2 a} \mathcal{E}_{k+r-2 a, r}^{s}$ has polynomial growth at the cusps, we have uniform convergence when Ret is large enough and we can complete the unfolding as usual. Then the last integral becomes

$$
\int_{0}^{\infty} \int_{0}^{1} E_{2 a}(z) \mathcal{E}_{k+r-2 a, r}^{s}(z) y^{t+k+r-2} e^{-2 \pi l y} e^{-2 \pi i l x} d x d y .
$$

To complete the computation we need the Fourier expansion of the completed version of $E_{m}(s)(z)$ as given in Prop. 11.2.16 of [4] (or Th. 3.1 of [6]), for integer $s$ with $s+r-a+k / 2>0$ which will be the case that interests us. It gives

$$
\begin{aligned}
& \mathcal{E}_{k+r-2 a, r}^{s}(z)=y^{a-r-\frac{k}{2}} E_{k-2 a}\left(s+r-a+\frac{k}{2}\right)(z)= \\
& \pi^{s+\frac{k}{2}+r-a} \Gamma\left(s+\frac{k}{2}+r-a+\frac{k-2 a}{2}\right)^{-1} \zeta(2 s+k-2 a-1+2 r)^{-1}\left\{y^{a-r-\frac{k}{2}} a_{0}(y)+\right. \\
& \quad \sum_{n \geq 1}\left(\frac{\sigma_{2 s+k+2 r-2 a-1}(n)}{n^{s+r-a+\frac{k}{2}}}\left(\sum_{a-\frac{k}{2} \leq j \leq s-1+\frac{k}{2}+r-a} \alpha_{j}^{+}(4 \pi n y)^{-j}\right) e^{-2 \pi n y} y^{a-r-\frac{k}{2}}\right) e^{2 \pi i n x} \\
& \left.+\sum_{n \leq-1}\left(\frac{\sigma_{2 s+k+2 r-2 a-1}(|n|)}{|n|^{s+r-a+\frac{k}{2}}}\left(\sum_{\frac{k}{2}-a \leq j \leq s-1+\frac{k}{2}+r-a} \alpha_{j}^{-}(4 \pi|n| y)^{-j}\right) e^{2 \pi n y} y^{a-r-\frac{k}{2}}\right) e^{2 \pi i n x}\right\} .
\end{aligned}
$$

Here

$$
a_{0}(y)=\left|\frac{k}{2}-a\right| !\left(\left(\begin{array}{c}
s+r-a+\frac{k}{2}-1+\left|\frac{k}{2}-a\right| \\
\left|\frac{k}{2}-a\right|
\end{array}\right) \Lambda(2 s+2 r-2 a+k) y^{s+r-a+\frac{k}{2}}+\right.
$$




$$
\left.\left(\begin{array}{c}
\left|\frac{k}{2}-a\right|-s-r+a-\frac{k}{2} \\
\left|\frac{k}{2}-a\right|
\end{array}\right) \Lambda(2-2 s-2 r+2 a-k) y^{1-s-r+a-\frac{k}{2}}\right)
$$

where $\Lambda(s):=\pi^{-s / 2} \Gamma(s / 2) \zeta(s)$ and

$$
\alpha_{j}^{ \pm}:=(-1)^{j}\left(j+\frac{|k-2 a|}{2}\right) !\left(\begin{array}{c}
s+r-a+\frac{k}{2}-1+\frac{|k-2 a|}{2} \\
j+\frac{|k-2 a|}{2}
\end{array}\right)\left(\begin{array}{c} 
\pm \frac{k-2 a}{2}-s-r+a-\frac{k}{2} \\
j \pm \frac{k-2 a}{2}
\end{array}\right) .
$$

Here $\left(\begin{array}{l}a \\ b\end{array}\right)$ with $a<0$ are defined in accordance with the convention that, if $a<0$ and $j \geq 0$, then $\left(\begin{array}{c}a+j \\ j\end{array}\right)=(a+j)(a+j-1) \ldots(a+1) / j !$

Therefore the coefficient of $e^{2 \pi i l x}(l>0)$ in the Fourier expansion of $E_{2 a}(z) \mathcal{E}_{k+r-2 a, r}^{s}(z)$ is

$$
\sum_{n \leq l} b_{n}(y) \sigma_{2 a-1}(l-n) e^{-2 \pi(l-n) y}
$$

where, if $n \neq 0$,

$$
\begin{gathered}
b_{n}(y):=\frac{\sigma_{2 s+k+2 r-2 a-1}(|n|)}{|n|^{s+r-a+\frac{k}{2}}}\left(\sum_{\substack{\operatorname{sgn}(n)\left(a-\frac{k}{2}\right) \leq j \leq s-1+\frac{k}{2}+r-a \\
b_{0}(y):=y^{a-r-k / 2} a_{0}(y) .}} \alpha_{j}^{\operatorname{sgn}(n)}(4 \pi|n| y)^{-j}\right) e^{-2 \pi|n| y} y^{a-r-\frac{k}{2}} \text { and }
\end{gathered}
$$

Also, for convenience, we set $\sigma_{2 a-1}(0):=-B_{2 a} /(4 a)$.

Therefore, with (13) we have that for $\operatorname{Re} t \gg 0$,

$$
\left\langle E_{2 a} \mathcal{E}_{k+r-2 a, r}^{s}, P_{l}^{t}\right\rangle_{k+r}=\int_{0}^{\infty} e^{-2 \pi l y}\left(\sum_{n \leq l} b_{n}(y) \sigma_{2 a-1}(l-n) e^{-2 \pi(l-n) y}\right) y^{t+k+r-2} d y .
$$

Since the finitely many terms corresponding to $b_{n}(y)$ with $n \geq 0$ can be directly evaluated at $t=0$ to give rational linear combinations of powers of $\pi$ and $i$, we will focus on the infinitely many terms indexed by $n<0$.

$$
\begin{aligned}
& \int_{0}^{\infty} e^{-2 \pi l y}\left(\sum_{n<0} b_{n}(y) \sigma_{2 a-1}(l-n) e^{-2 \pi(l-n) y}\right) y^{t+k+r-2} d y \\
= & \sum_{\frac{k}{2}-a \leq j \leq s-1+\frac{k}{2}+r-a} \alpha_{j}^{-}(4 \pi)^{-j} \sum_{n<0} \frac{\sigma_{2 s+k+2 r-2 a-1}(|n|) \sigma_{2 a-1}(l-n)}{|n|^{s+r-a+\frac{k}{2}+j}} \int_{0}^{\infty} e^{-4 \pi(l-n) y} y^{\frac{k}{2}+a-j-1+t} \frac{d y}{y} \\
= & \sum_{\frac{k}{2}-a \leq j \leq s-1+\frac{k}{2}+r-a} \alpha_{j}^{-}(4 \pi)^{1-\frac{k}{2}-a-t} \Gamma\left(\frac{k}{2}+a-j-1+t\right) \sum_{n>0} \frac{\sigma_{2 s+k+2 r-2 a-1}(n) \sigma_{2 a-1}(l+n)}{n^{s+r-a+\frac{k}{2}+j}(n+l)^{\frac{k}{2}+a-j-1+t}}
\end{aligned}
$$

Since $j \leq s-1+\frac{k}{2}+r-a$ we can expand binomially the term $((n+l)-l)^{s+\frac{k}{2}+r-a-1-j}$. This, together with the trivial identity $\sigma_{w}(n)=n^{w} \sigma_{-w}(n)$, imply that the last sum of (16) becomes

$$
\begin{gathered}
\sum_{n>0} \frac{\sigma_{-2 s-k-2 r+2 a+1}(n) n^{s+\frac{k}{2}+r-a-1-j} \sigma_{2 a-1}(l+n)}{(n+l)^{\frac{k}{2}+a-j-1+t}}=\sum_{\mu=0}^{s+\frac{k}{2}+r-a-1-j}\left(\begin{array}{c}
s+\frac{k}{2}+r-a-1-j \\
\mu
\end{array}\right) \times \\
(-l)^{s+\frac{k}{2}+r-a-1-j-\mu} D_{l}\left(2 a-1,-2 s-k-2 r+2 a+1 ; \frac{k}{2}+a-j-1+t-\mu\right)
\end{gathered}
$$


where

$$
D_{l}(\alpha, \beta ; w):=\sum_{n>l} \frac{\sigma_{\alpha}(n) \sigma_{\beta}(n-l)}{n^{w}} .
$$

In view of (11), we aim to investigate the series in (17) at $t=0$. We are mainly interested in the case that least one of $s+k+r-1$ and $s+k+r-2 a$ is outside the critical strip. In this case, the series $D_{l}$ appearing in (17) are not in the initial region of convergence when $t=0$. However, this convolution series has been studied by M. Kiral in [8] and has given the analytic continuation. Here we do not need the full analytic continuation established in [8] but rather a partial extension. Since [8] has not appeared in print yet, we give here a proof of the part of the analytic continuation we need for our purposes.

For $r$ odd, set

$$
s=1, \quad a=\frac{k+r-1}{2} .
$$

These values of $s$ and $a$ satisfy the conditions $s+r-a+k / 2>0$ and $a, b=(k-2 a+2 r+2) / 2 \geq 2$ that are required for our construction. Further, with these values of $s, a$, the parameter $j$ in (16) ranges between $(1-r) / 2$ and $(1+r) / 2$. For each of these values of $j$, the parameter $\mu$ in (17) ranges between 0 and $\frac{1+r}{2}-j$ and thus $j+\mu$ ranges between $(1-r) / 2$ and $(1+r) / 2$.

Therefore, we need to show all shifted convolutions

$$
D_{l}(k+r-2,-r-2 ; \nu+t) ; \quad(\nu=k-2, \ldots, k-2+r)
$$

appearing in (17) have an analytic continuation in a neighbourhood of $t=0$.

To achieve that, we first prove the following lemma, which is a slight generalisation of Prop. 6 of [8].

Lemma 4.1. Let $l \in \mathbb{N}_{0}, \alpha \in \mathbb{Z}$ and $\beta<-1$. Then for each $w$ with $\operatorname{Re}(w) \gg 0$, we have

$$
D_{l}(\alpha, \beta ; w)=\zeta(1-\beta) \sum_{m=1}^{\infty} m^{\beta-1} \sum_{\substack{x \bmod m \\(x, m)=1}} e^{\frac{-2 \pi i x l}{m}} E_{l}\left(w, \alpha ; \frac{x}{m}\right)
$$

where $E_{l}\left(s, \alpha ; \frac{x}{m}\right)$ the (truncated) Estermann zeta function, defined, for $\operatorname{Re}(s) \gg 0$, by

$$
E_{l}\left(s, \alpha ; \frac{x}{m}\right):=\sum_{n>l} \frac{\sigma_{\alpha}(n) e^{2 \pi i \frac{x n}{m}}}{n^{s}} \quad s, \alpha \in \mathbb{C}, x / m \in \mathbb{Q} .
$$

Proof. The series $D_{l}(\alpha, \beta ; w)$ is absolutely convergent for $\operatorname{Re}(w) \gg 0$. We apply the Ramanujan identity

$$
\sigma_{\beta}(n)=\zeta(1-\beta) \sum_{m=1}^{\infty} m^{\beta-1} \sum_{\substack{x \bmod m \\(x, m)=1}} e^{\frac{2 \pi i x n}{m}}
$$

with $n-l$ instead of $n$ to obtain:

$$
\begin{aligned}
D_{l}(\alpha, \beta ; w) & =\sum_{n>l} \frac{\sigma_{\alpha}(n)}{n^{w}} \zeta(1-\beta) \sum_{m=1}^{\infty} m^{\beta-1} \sum_{\substack{x \bmod m \\
(x, m)=1}} e^{\frac{2 \pi i x(n-l)}{m}} \\
& =\zeta(1-\beta) \sum_{m=1}^{\infty} m^{\beta-1} \sum_{\substack{x \bmod m \\
(x, m)=1}} e^{\frac{-2 \pi i x l}{m}} \sum_{n>l} \frac{\sigma_{\alpha}(n) e^{\frac{2 \pi i x n}{m}}}{n^{w}}
\end{aligned}
$$


as asserted.

This lemma gives the meromorphic continuation of $D_{l}(k+r-2,-r-2 ; \nu+t)$ through the meromorphic continuation of the (full) Estermann zeta function $E\left(s, \alpha ; \frac{x}{m}\right):=E_{0}\left(s, \alpha ; \frac{x}{m}\right)$. Specifically, Lemma 18, in our setting, gives

$$
D_{l}(k+r-2,-r-2 ; \nu+t)=\zeta(3+r) \sum_{m=1}^{\infty} m^{-3-r} \sum_{\substack{x \bmod m \\(x, m)=1}} e^{\frac{-2 \pi i x l}{m}} E_{l}\left(\nu+t, k+r-2 ; \frac{x}{m}\right) .
$$

Now, each $E\left(\nu+t, k+r-2 ; \frac{x}{m}\right)$ can be expressed in terms of Hurwitz zeta functions $\zeta(s, x)$ as follows, for $\operatorname{Re}(w) \gg 0$ :

$$
\begin{aligned}
E\left(\nu+t, k+r-2 ; \frac{x}{m}\right) & =\sum_{n>0} \frac{\left(\sum_{d \mid n} d^{k+r-2}\right) e^{2 \pi i \frac{x n}{m}}}{n^{\nu+t}}=\sum_{d, n>0} \frac{e^{2 \pi i \frac{x d n}{m}}}{d^{\nu+t-k-r+2} n^{\nu+t}} \\
& =\sum_{d_{1}, n_{1}>0} \sum_{u, v=1}^{m} \frac{e^{2 \pi i \frac{x\left(m d_{1}+u\right)\left(m n_{1}+v\right)}{m}}}{\left(m d_{1}+u\right)^{\nu+t-k-r+2}\left(m n_{1}+v\right)^{\nu+t}} \\
& =m^{k+r-2 \nu-2 t-2} \sum_{u, v=1}^{m} e^{2 \pi i \frac{x u v}{m}} \zeta\left(-k-r+2+\nu+t, \frac{u}{m}\right) \zeta\left(\nu+t, \frac{v}{m}\right) .
\end{aligned}
$$

It is well-known that $\zeta(s, x)$ has a meromorphic continuation to $\mathbb{C}$, with only a simple pole at $s=1$. Therefore the RHS is analytic in a neighbourhood of $t=0$, since $\nu \in\{k-2, \ldots, k-$ $2+r\}$, thus giving the analytic continuation the LHS in this neighbourhood. Further, when $x \in(0,1), \zeta(\nu+t, x)$ is bounded by a constant independent of $x$, since $\nu+\operatorname{Re}(t)>1$. By the functional equation of $\zeta(s, x)$ (or, in the case $\nu=k+r-2$, the Taylor expansion of $\zeta(s, x+1)$ at $x=0$ ), we deduce that, for $t$ in a small neighbourhood of 0

$$
\zeta\left(-k-r+2+\nu+t, \frac{u}{m}\right) \ll_{k, r} m^{t}+1
$$

Therefore, with (20),

$$
E\left(\nu+t, k+r-2 ; \frac{x}{m}\right) \ll_{k, r} m^{k+r-2 \nu-t}+m^{k+r-2 \nu-2 t} .
$$

Applying this bound to the series of the RHS of (19), we see that each term is holomorphic in a neighbourhood of $t=0$ and bounded by $m^{k-2 \nu-t-1}+m^{k-2 \nu-2 t-1}$. Therefore, the function $D_{l}(k+r-2,-r-2 ; \nu+t)$ is holomorphic at $t=0$ for all $\nu \in\{k-2, \ldots, k-2+r\}$.

For $r$ even, set

$$
s=1, \quad a=\frac{k+r}{2} .
$$

In this case, we need to show that the meromorphic continuation of $D_{l}(k+r-1,-r-1 ; \nu+t)$ is holomorphic at $t=0$ for all $\nu \in\{k-1, \ldots, k-1+r\}$. We do that by working in exactly the same way as above, employing Lemma 18 and the following analogue of (20):

$$
E\left(\nu+t, k+r-1 ; \frac{x}{m}\right)=m^{k+r-2 \nu-2 t-1} \sum_{u, v=1}^{m} e^{2 \pi i \frac{x u v}{m}} \zeta\left(-k-r+1+\nu+t, \frac{u}{m}\right) \zeta\left(\nu+t, \frac{v}{m}\right) .
$$


Proof of Theorem 1.1: As mentioned above, the terms corresponding to non-negative $n$ in the sum in $(15)$ lead to elements of $\mathbb{Q}(\pi, i)$.

Let $r$ be odd. What we just proved, together with (9) then imply that the $l$-th Fourier coefficient of $\mathbf{E}_{k+r}^{*}(z, 2)$ belongs to the field

$$
\mathcal{D}_{r}=\mathbb{Q}\left(\pi, i, D_{l}(k+r-2,-r-2 ; k-2), \ldots, D_{l}(k+r-2,-r-2 ; k-2+r)\right)
$$

obtained by adjoining to $\mathbb{Q}(\pi, i)$ the values of the shifted convolution $D_{l}(k+r-2,-r-2 ; n)$ at $n=k-2, \ldots k-2+r$.

On the other hand, (3) implies that

$$
\mathbf{E}_{k+r}^{*}(z, 2)=\mathbf{E}_{2}^{*}(z, k+r) .
$$

Therefore, with Proposition 2.2 for $s_{0}=2$ and odd $r^{\prime} \geq 1$

$$
\frac{L_{f}^{*}(k+r)}{L_{f}^{*}\left(k+r^{\prime}\right)} \in \mathcal{D}_{r} \mathcal{D}_{r^{\prime}} \mathbb{Q}(f)
$$

Setting $r^{\prime}=1$, we deduce the first case of Theorem 1.1 .

Let $r$ be even. In the same way as above we find that the $l$-th Fourier coefficient of $\mathbf{E}_{k+r}^{*}(z, 1)=\mathbf{E}_{1}^{*}(z, k+r)$ belongs to the field

$\mathbb{Q}\left(\pi, i, D_{l}(k+r-1,-r-1 ; k-1), D_{l}(k+r-1,-r-1 ; k), \ldots, D_{l}(k+r-1,-r-1 ; k-1+r)\right)$

Applying Proposition 2.2 with $s_{0}=1$, we deduce, for even $r^{\prime}>1$,

$$
\frac{L_{f}^{*}(k+r)}{L_{f}^{*}\left(k+r^{\prime}\right)} \in \mathcal{D}_{r} \mathcal{D}_{r^{\prime}} \mathbb{Q}(f)
$$

where $\mathcal{D}_{r}$ denotes the field obtained by adjoining to $\mathbb{Q}(\pi, i)$ the values of the shifted convolution $D_{l}(k+r-1,-r-1 ; n)$ at $n=k-1, \ldots k-1+r$. Setting $r^{\prime}=2$, we deduce the second case of Theorem 1.1.

\section{References}

[1] A. A. Beilinson, Higher regulators of modular curves. Applications of algebraic K-theory to algebraic geometry and number theory (Contemporary Mathematics 5 (1986)), 1-34

[2] F. Brown Modular Values and the relative completion of the fundamental group of M1,1 arXiv:1407.5167

[3] F. Brunault Valeur en 2 de fonctions $L$ de formes modulaires de poids 2 : théorème de Beilinson explicite. Bulletin de la Société Mathmatique de France 135 (2007), no 2, pp. 215-246.

[4] H. Cohen, F. Strömberg Modular Forms: A Classical Approach, American Mathematical Society (2017) 
[5] P. Deligne Valeurs de Fonctions L et périodes d'intégrales, Proceedings of Symposia in Pure Mathematics 33, 313-346 (1979).

[6] N. Diamantis and C. O'Sullivan Kernels of L-functions of cusp forms Math. Annalen, 346, (2010), no. 4, 897 - 929

[7] N. Diamantis and C. O'Sullivan Kernels for products of L-functions Algebra and Number Theory, 7 (2013), no. 8, 1883 - 1917.

[8] M. Kiral Shifted Divisor Sum Dirichlet Series preprint

[9] N. Koblitz Non-integrality of the periods of cusp forms outside the critical strip. Funkcional. Anal. i Prilozhen. 9, 52-55 (1975)

[10] Y. Manin Periods of cusp forms, and p-adic Hecke series. Mat. Sb. (N.S.), 21(134):371$393(1973)$

[11] Y. Manin Iterated integrals of modular forms and non-commutative modular symbols Algebraic geometry and number theory, 565-597, Prog. Math. 253 (2006)

[12] Y. Manin Iterated Shimura integrals Moscow Math. J. 5 (2005), 869-881

[13] M. Rogers, W. Zudilin From L-series of elliptic curves to Mahler measures, Compos. Math. 148 (2012), no. 2, p. 385-414.

[14] G. Shimura The special values of the zeta functions associated with cusp forms Comm. Pure Appl. Math., 29(6):783-804 (1976)

[15] M. Kontsevich, D. Zagier Periods. Mathematics Unlimited-2001 and Beyond (B. Engquist and W. Schmid, eds.), Springer, Berlin-Heidelberg-New York (2001), 771-808

[16] V. Pasol, A. Popa Modular forms and period polynomials. Proc. Lond. Math. Soc. 107/4 (2013), 713-743

[17] D. Zagier Modular forms whose Fourier coefficients involve zeta-functions of quadratic fields in Modular Functions of One Variable VI, Lecture Notes in Math. 627, SpringerVerlag, Berlin-Heidelberg-New York (1977) 105-169 